\title{
Point-of-Care Toolkit for Multiplex Molecular Diagnosis of SARS- CoV-2 and Influenza A and B Viruses
}

\author{
Dohwan Lee, Chia-Heng Chu, and A. Fatih Sarioglu*
}

Cite This: ACS Sens. 2021, 6, 3204-3213

Read Online

ABSTRACT: Severe acute respiratory syndrome-coronavirus 2 (SARS$\mathrm{CoV}-2$ ) is still spreading around the globe causing immense public health and socioeconomic problems. As the infection can progress with mild symptoms that can be misinterpreted as the flu, self-testing methods that can positively identify SARS-CoV-2 are needed to effectively track and prevent the transmission of the virus. In this work, we report a point-ofcare toolkit for multiplex molecular diagnosis of SARS-CoV-2 and influenza A and B viruses in saliva samples. Our assay is physically programmed to run a sequence of chemical reactions on a paper substrate and internally generate heat to drive these reactions for an autonomous extraction, purification, and amplification of the viral RNA. Using our assay, we could reliably detect SARS-CoV-2 and influenza viruses at

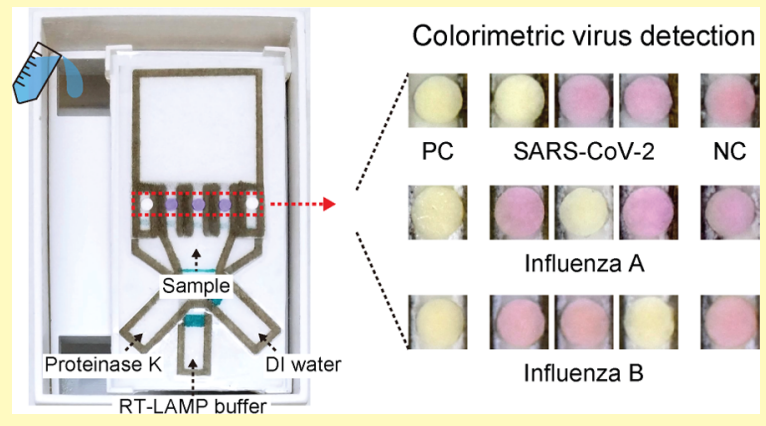
concentrations as low as 50 copies $/ \mu \mathrm{L}$ visually from a colorimetric analysis. The capability to autonomously perform a traditionally labor-intensive genetic assay on a disposable platform will enable frequent, on-demand self-testing, a critical need to track and contain this and future outbreaks.

KEYWORDS: SARS-CoV-2, influenza virus, multiplexed molecular diagnosis, point-of-care RNA test, programmed paper microfluidics, isothermal PCR amplification

S ince the first report of atypical pneumonia cases in Wuhan, China, at the end of 2019, the causative agent has been identified as a new betacoronavirus named severe acute respiratory syndrome-coronavirus 2 (SARS-CoV-2). ${ }^{1-6}$ Due to its rapid spread around the globe, the World Health Organization (WHO) declared the coronavirus disease 2019 (COVID-19) a pandemic on 11 March $2020 .^{1-6}$ Besides its efficient transmission, a challenging aspect of the disease is that it could be asymptomatic or its symptoms could easily be interpreted as due to flu complicating identification of people infected with the virus. ${ }^{5-10}$ In fact, the Center for Disease Control and Prevention recently emphasized the importance of differentiating between SARS-CoV-2, influenza $A$, and influenza $B$ viruses, all of which lead to similar respiratory symptoms. ${ }^{11}$ As such, there is an urgent need for tests that can positively identify COVID-19 cases to enable timely response.

SARS-CoV-2 can currently be detected by molecularly amplifying the virus RNA sampled through a nasal swab or from saliva as the reverse transcription-quantitative polymerase chain reaction (RT-qPCR) kit is, in fact, widely used to diagnose COVID-19. ${ }^{7-10}$ Although RT-qPCR is a sensitive and accurate method, it has several limitations to be employed in decentralized settings. First, the process typically employs a bulky and expensive thermal cycler, leading to a prolonged amplification process. ${ }^{12-17}$ While miniaturized RT-qPCR approaches using handheld instruments ${ }^{18-20}$ could accelerate the amplification process, these techniques still rely on external equipment for sample preparation and/or data analysis which can potentially hinder their use on-site. Moreover, the RTqPCR is vulnerable to inhibitors such as body fluids (blood, urine, sweat, etc.), which demands painstaking steps for highpurity RNA extraction by trained technicians with sophisticated laboratory instruments. ${ }^{21-24}$ Direct RT-qPCR methods that can skip RNA extraction steps ${ }^{25,26}$ can be used to simplify the process but at the expense of reduced sensitivity. ${ }^{27}$ On the other hand, tracking the transmission of a virus with nonspecific symptoms requires frequent self-testing with simple-to-use, standalone kits that can perform molecular amplification in a point-of-care (POC) assay setting.

Isothermal polymerase chain reaction (PCR) techniques that amplify a specific region of an RNA without the need for thermocycling are attractive for developing POC molecular tests. $^{12-17}$ The reverse transcription loop-mediated isothermal amplification (RT-LAMP) is a particularly robust and highly sensitive technique with rapid response. ${ }^{21-24}$ The RT-LAMP employs four to six primers to specifically amplify target RNA

Received: April 6, 2021

Accepted: August 24, 2021

Published: September 15, 2021 


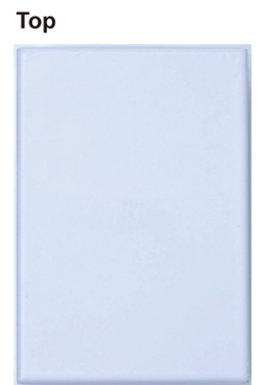

Top cover

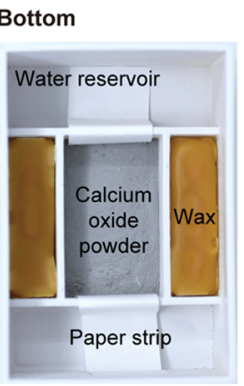

Internal Heat module (IH module) b

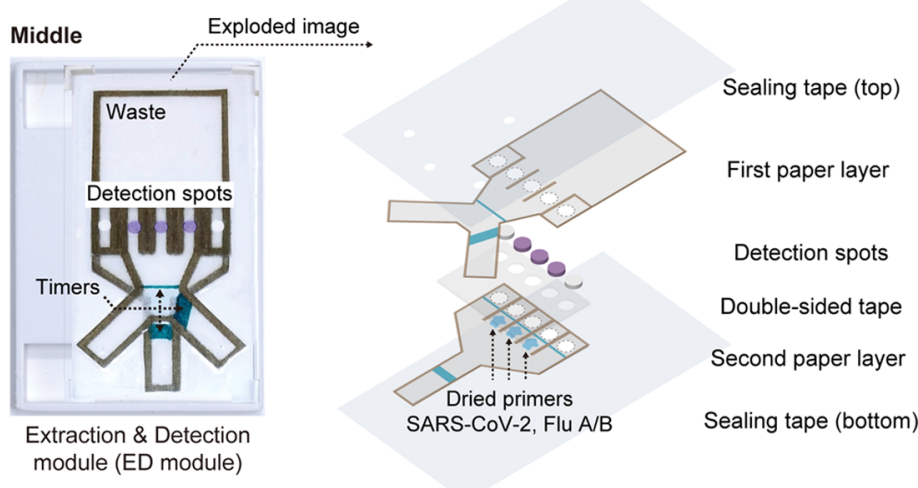

c
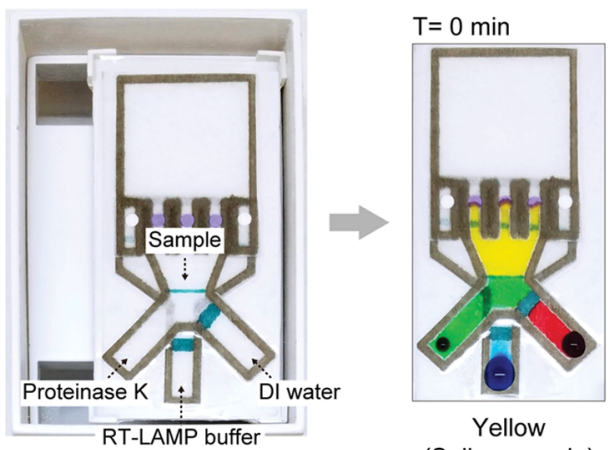

(Saliva sample)

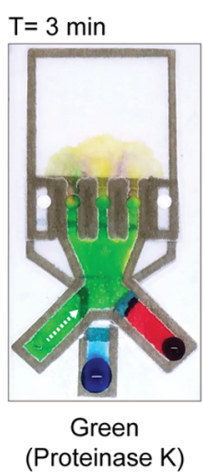

(Proteinase K)

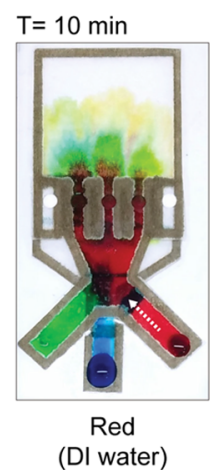

(DI water)

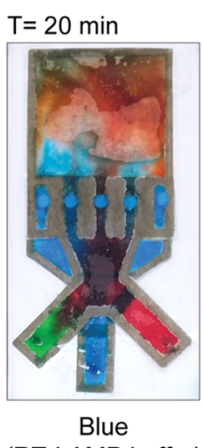

(RT-LAMP buffer)

d

1. Seal inlet holes with tape

2. Pour water for internal heating

3. Wait 1 hour for RT-LAMP reaction

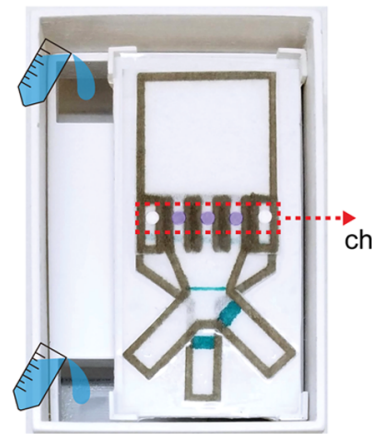

Figure 1. Design of the assay and its operation. (a) Photographs showing components of the developed POC toolkit. The top cover is a plastic cover for the thermal isolation of the running assay. The internal heat module (IH module) consists of several reservoirs filled with $\mathrm{CaO}$ powder, water, and wax. A cellulose paper strip is placed between the $\mathrm{CaO}$ and water reservoirs to carry the water to the $\mathrm{CaO}$ reservoir. The extraction and detection module (ED module) is a multilayered paper platform. The flow paths for manipulating different reagents are defined by imprinting on the paper with a water-insoluble ink (brown). Timers were imprinted (green) on the flow paths to introduce controlled amounts of delay to the flow of different reagents. (b) Exploded image of the ED module showing individual layers. Two paper layers imprinted with features were laminated in between polymer tapes and were coupled through the detection spots punched out of filter paper pretreated with chemicals for RNA extraction. The primer mixtures specifically targeting SARS-CoV-2 and influenza A and B viruses were dried in front of the detection spots to be carried by the RT-LAMP buffer flow on the second paper layer. (c) Time-lapse images of the ED module showing its capability to coordinate the delivery of four dye solutions simultaneously loaded into the module. The dye solutions were used instead of the actual sample and reagents for visual investigation. The images show the state of the module at selected timepoints: the delivery of the saliva sample (yellow), proteinase $\mathrm{K}$ (green), DI water (red), and RT-LAMP buffer (blue) to the detection spots. (d) Schematic showing the procedure to operate the developed assay. The presence of SARS-CoV-2 and influenza A and B viruses in the processed sample can be visually identified by the color changes in the corresponding detection spot.

sequences at $60-72{ }^{\circ} \mathrm{C}$ even in the presence of the inhibitors. ${ }^{16,17}$ Furthermore, the Bst polymerase used in the RT-LAMP is highly resistant to high concentrations of inhibitory substances. ${ }^{2-24}$ Also, because the Bst polymerase exhibits strong reverse transcription activity at elevated temperatures up to $72{ }^{\circ} \mathrm{C}$, the RT-LAMP does not require an additional enzyme for reverse transcription of viral RNA to cDNA. $^{23,28}$ While the RT-LAMP was successfully performed on a paper substrate $e^{24,28-34}$ or on a miniaturized device ${ }^{35-37}$ together with colorimetric detection of amplicons, these attempts fell short of achieving a standalone, sample-to-answer 
POC assay as the RNA extraction and amplification still required human intervention or external instruments.

Here, we report a fully integrated POC toolkit for multiplex molecular diagnosis of SARS-CoV-2 and influenza A and B viruses from saliva. To extract, amplify, and detect viral RNAs, we designed a paper-based device with a built-in capillary flow regulation, enabled by timers imprinted on flow paths to produce controlled delays. The controlled routing of the capillary flow on paper enabled multiple reagents to be sequentially delivered to the assigned detection spots, providing a mechanism for automated extraction of the viral RNA and also a timely introduction of reaction buffer for amplification. The colorimetric RT-LAMP was then automatically performed on all detection spots at a fixed temperature maintained by a calibrated exothermic reaction. The presence of any of the SARS-CoV-2 and influenza A and B viruses in the sample was then exposed through color changes in the corresponding detection spots.

\section{RESULTS AND DISCUSSION}

Assay Design and Operation. Our assay is composed of a multilayered paper platform for RNA extraction, amplification, and colorimetric detection (ED module) sandwiched between an internal heat module ( $\mathrm{IH}$ module) for driving the RT-LAMP process at the bottom and a top cover for thermal isolation (Figure 1a). To generate heat within the assay, the $\mathrm{IH}$ module contains calcium oxide $(\mathrm{CaO})$, which reacts with water in an exothermic reaction, and a peripheral wax that functions as a temperature regulator by melting at the desired temperature. The exothermic reaction is triggered through a cellulose strip that carries water from a reservoir at a fixed rate to an otherwise isolated $\mathrm{CaO}$. The chemical reactions are carried out on the paper component (ED module), which routes multiple capillary flow streams to sequentially introduce reagents to the detection spot for RNA extraction and RTLAMP reaction. The flow paths on the paper were created by simply imprinting with different types of water-insoluble inks when defining channel boundaries or timers to delay flow for a preset duration.

The 3D capillary flow network in the assay was established through stacked layers of paper laminated in between polymer tapes (Figure 1b). The tape at the top was patterned to create inlets for the sample and RNA extraction reagents. The first paper layer was designed to collect these reagents and deliver them to the sample in sequence with set delays in between. The detection spots were cutouts from a commercial filter paper pretreated with chemicals for cell lysis and preservation of the extracted nucleic acid and were positioned to receive flow from paper layers both above and below. Among those five detection spots, three central ones (purple) were used for extraction and detection of viral RNA from SARS-CoV-2 and influenza A and B viruses, respectively. Two peripheral spots (white) were pretreated to serve as positive (PC) and negative control (NC). The detection spots were also coupled to a second paper layer below, designed to release the RT-LAMP buffer once the viral RNA is extracted. A double-sided tape between the two paper layers not only held them together but also ensured the RNA extraction and the RT-LAMP reagents remained isolated except on the detection spots. The components that gave our assay the multiplex detection capability were the dried primer mixtures specifically targeting SARS-CoV-2 and influenza A and B viruses deposited in front of the detection spots to be carried by the RT-LAMP buffer flow.

Our assay was physically programmed via features imprinted on paper for automated sequential execution of a set of chemical processes to extract the viral RNA. We constructed the fluidic circuit on paper using two water-insoluble inks with differing affinities to the laminating polymer tape: one (brown) that permanently adhered to the polymer tape to define the flow boundaries and another (green) that gradually delaminated from the polymer tape when wetted to create delay lines (timers) on the flow paths at strategically chosen nodes. Desired delays between flows of different reagents were set by tuning the width of the timers (see the Materials and Methods). We optimized the assay geometry to achieve the desired reagent flow sequence with target incubation times through testing with dye solutions (Figure 1c). In the optimized design, the deposited $20 \mu \mathrm{L}$ sample (represented by yellow dye) was funneled toward the detection spots as a thin timer blocked the backflow until the next reagent arrived. Proteinase $\mathrm{K}$ solution (green, $30 \mu \mathrm{L}$ ) reached the detection spots in $3 \mathrm{~min}$, while $60 \mu \mathrm{L}$ of deionized (DI) water for washing (red) took $10 \mathrm{~min}$ to surpass a thick timer. RT-LAMP buffer (blue, $100 \mu \mathrm{L}$ ), flowed on the paper layer below, arrived at the detection spots in $20 \mathrm{~min}$, a time duration we set for completing the RNA extraction process.

Once all reagents were delivered to the detection spots, the colorimetric RT-LAMP reaction was initiated by triggering the exothermic reaction in the $\mathrm{IH}$ module with water poured into the designated reservoir (Figure 1d). To minimize thermal loss and evaporation, the inlets were sealed with tape (Figure S1), and the device was covered for $1 \mathrm{~h}$ to complete the RT-LAMP. At the end of the process, amplicons from a specific gene of the target virus were detected from a change in the color of the detection spots (see the Materials and Methods). Among the three central detection spots, the one next to the PC spot targeted the SARS-CoV-2, while the middle spot and the next one screened for influenza A and B viruses, respectively. A saliva sample was only scored positive for any one of the three viruses if there was a color change (from pink to yellow) in the corresponding detection spot along with a color change in the $\mathrm{PC}$ spot and no change in $\mathrm{NC}$ spot color to ensure against artifacts.

Characterization and Optimization of Viral RNA Extraction. To determine the optimum substrate for building the detection spots in our assay, we compared the performance of commercially available RNA sampling papers. Because both RNA extraction and amplification were to be performed on the detection spots, in our experiments, we aimed to identify a medium that was not only efficient in extracting the virus RNA but also compatible with RT-LAMP process. We characterized three commercial nucleic acid sampling papers along with cellulose and nitrocellulose paper as controls using samples prepared by spiking inactivated SARS-CoV-2 intact virus particles into saliva samples collected from healthy donors under Georgia-Tech Institutional Review Board (IRB)approved protocol. To quantify the amount of viral RNA on each paper, we subjected $2.5 \mathrm{~mm}$-diameter discs punched out from papers to real-time RT-LAMP (see the Materials and Methods for details). We found that the FTA Elute card required the least time of amplification for a detectable RNA signal yielding the smallest time threshold $\left(T_{\mathrm{t}}\right)$ value of 24.9 min, while measured $T_{\mathrm{t}}$ values obtained from FTA card and RNASound card were 41.02 and $37.15 \mathrm{~min}$, respectively 
a

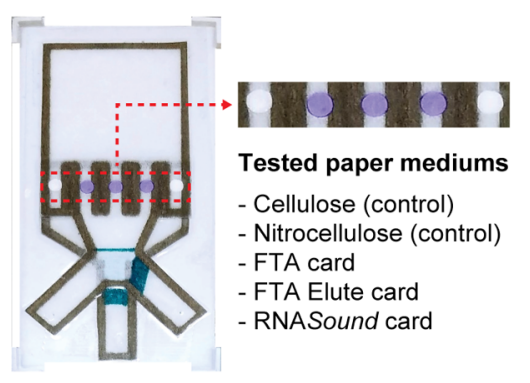

FTA card

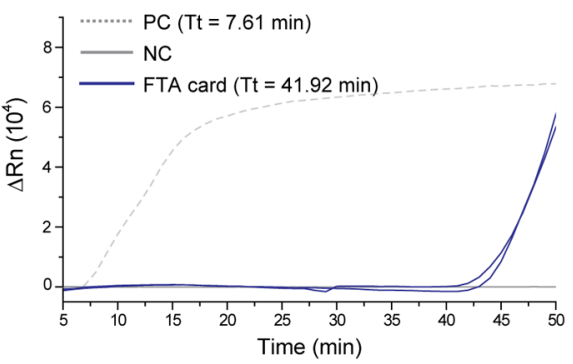

Cellulose

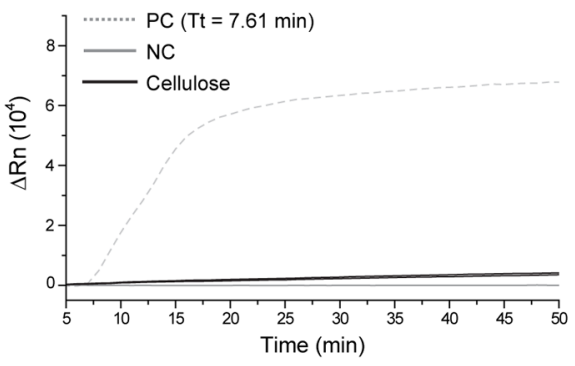

FTA Elute card

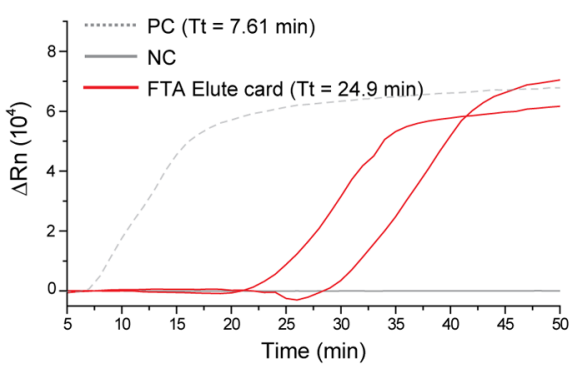

Nitrocellulose

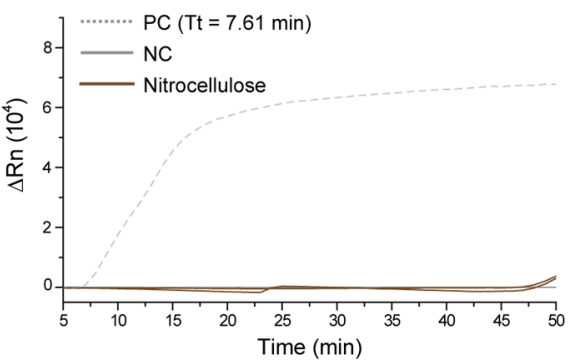

RNASound card

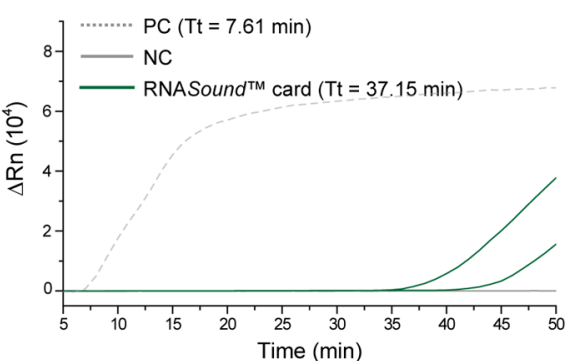

b

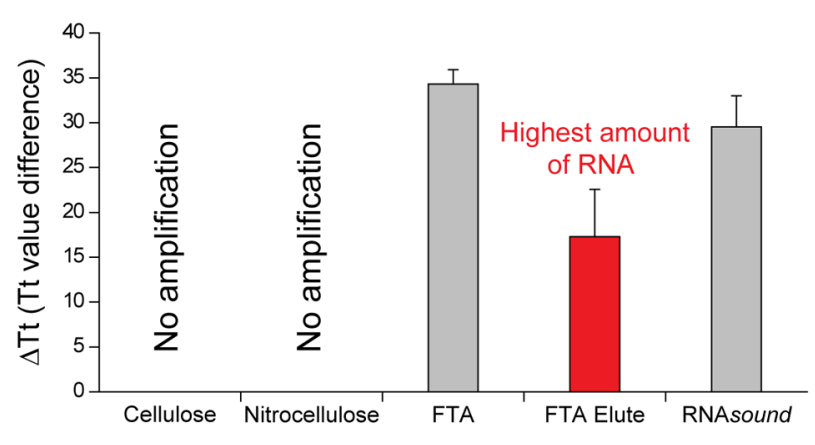

C

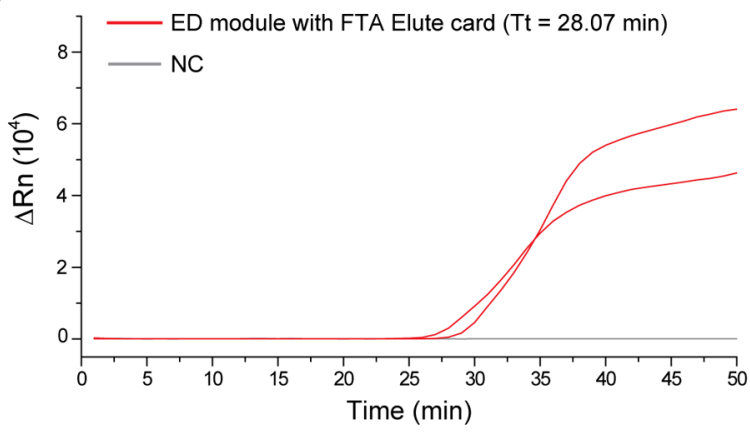

Figure 2. Characterization and optimization of viral RNA extraction. (a) Amplification plots from the real-time RT-LAMP of the extracted SARSCoV-2 on five different filter papers: cellulose and nitrocellulose paper as controls and three commercially available nucleic acid sampling papers. No amplifications were observed on the cellulose and nitrocellulose papers. Amplification occurred on the FTA, FTA Elute, and RNASound cards with different $T_{\mathrm{t}}$ values. For each amplification, measurements were performed in pairs to ensure against artifacts. (b) Measured mean $T_{\mathrm{t}}$ value differences $\left(\Delta T_{\mathrm{t}}\right)$ for different papers tested. The error bars represent the standard deviation $(n=3)$. (c) Measured amplification curve when the SARS-CoV-2 RNA was autonomously extracted by an ED module equipped with detection spots made out of the FTA Elute card and was subsequently subjected to real-time RT-LAMP outside of the device.

(Figure 2a). As anticipated, the cellulose and nitrocellulose papers did not show any amplification since neither of these incorporated chemicals needed for RNA extraction. Comparing the tested substrates based on $\Delta T_{\mathrm{t}}$ values, obtained by subtracting the $T_{\mathrm{t}}$ value from that of the PC, we concluded that the FTA Elute card could extract viral RNA with the highest efficiency and was also compatible with RT-LAMP reaction (Figure 2b).

Next, we tested the FTA Elute card when integrated into our assay as detection spots and investigated the RNA extraction performance. The paper device was programmed to automatically deliver the same set of reagents to the FTA Elute card spots. Once the SARS-CoV-2-spiked saliva samples were processed on the device, the extracted viral RNA was recovered by separating the spots from the device and amplified through real-time RT-LAMP. The results demonstrated successful extraction of SARS-CoV-2 RNA from the sample on the device (Figure 2c). Importantly, we measured the $T_{\mathrm{t}}$ value to be similar to those achieved by manual processing of the saliva sample with the FTA Elute card following the manufacturer's instructions (24.9 $\mathrm{min}$ vs 28.07 min), showing that the automated RNA extraction coordinated by the built-in flow control within the device was equally efficient.

Autonomous RNA Amplification and Colorimetric Detection. To amplify the extracted and purified viral RNA directly on the device without relying on an external process, we internally generated the heat needed to carry out the RTLAMP reaction through an exothermic reaction

$$
\begin{gathered}
\mathrm{CaO}(\mathrm{s})+\mathrm{H}_{2} \mathrm{O}(\mathrm{l}) \rightarrow \mathrm{Ca}(\mathrm{OH})_{2}(\mathrm{~s}) \\
\Delta H_{\mathrm{r}}=-65.2 \mathrm{~kJ} / \mathrm{mol}
\end{gathered}
$$

In a reservoir below the extracted $\mathrm{RNA}, \mathrm{CaO}$ powder was made to react with water that was continuously carried from the neighboring reservoir via a cellulose paper strip (Figure $3 a$ ). While the exothermic reaction produced sufficient heat, it failed to provide an isothermal environment with the device temperature overshooting to levels $\left(\sim 90{ }^{\circ} \mathrm{C}\right)$ that could inactivate the Bst polymerase (Figure $3 \mathrm{~b}$ ). In an attempt to regulate the device temperature at a level optimal for the RTLAMP reaction $\left(60-72{ }^{\circ} \mathrm{C}\right)$, we surrounded the reaction 


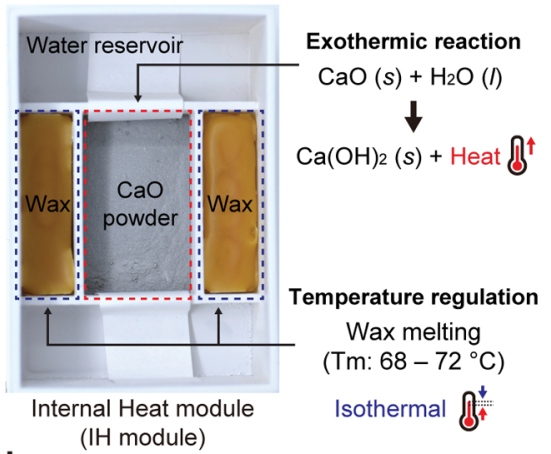

d

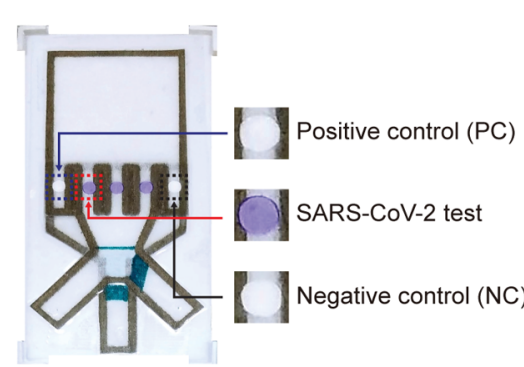

b

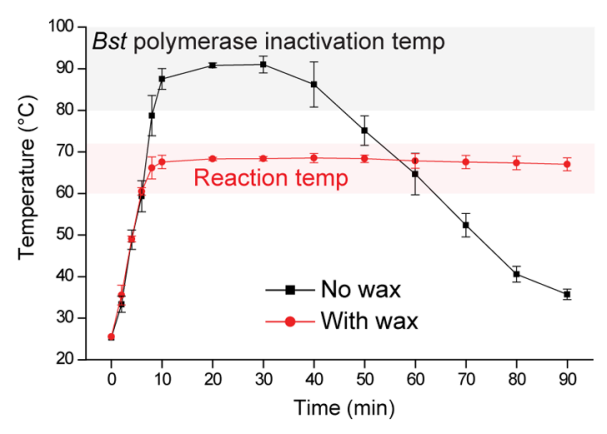

C
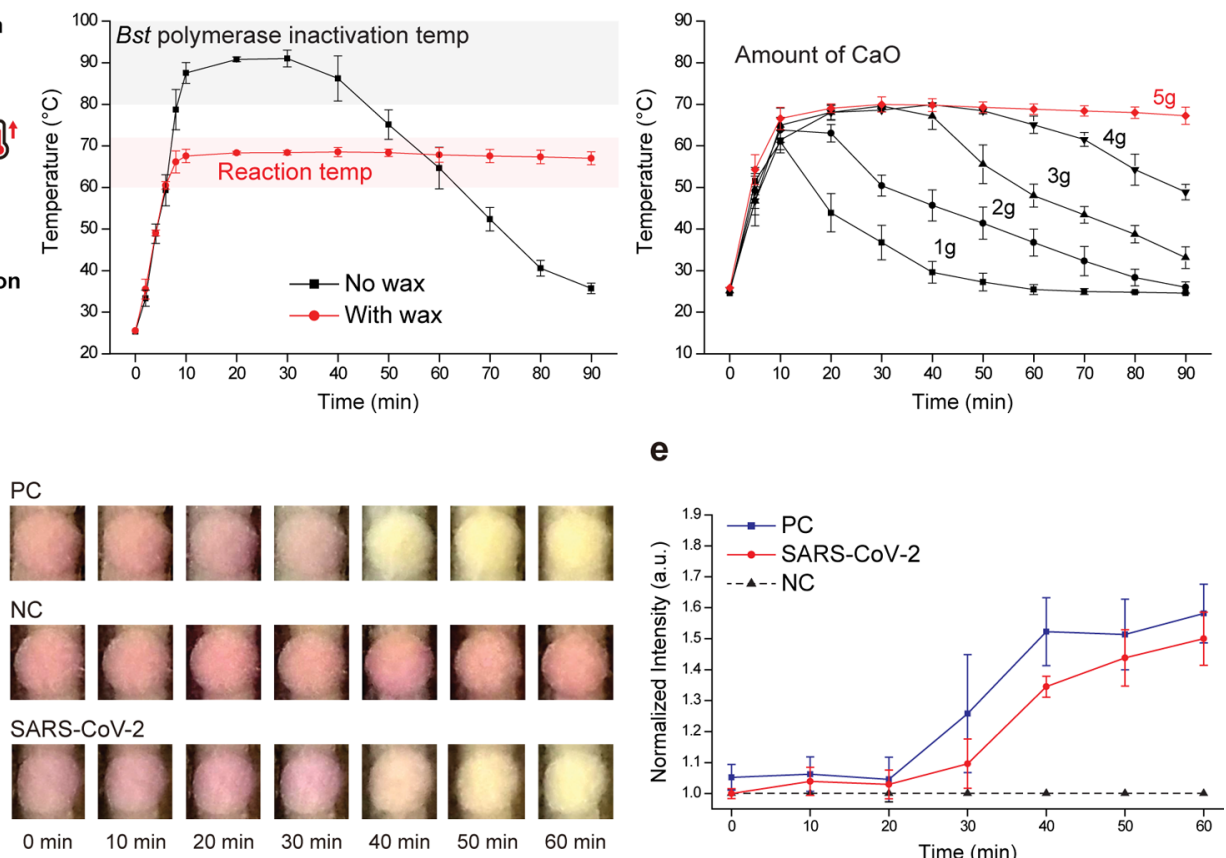

e

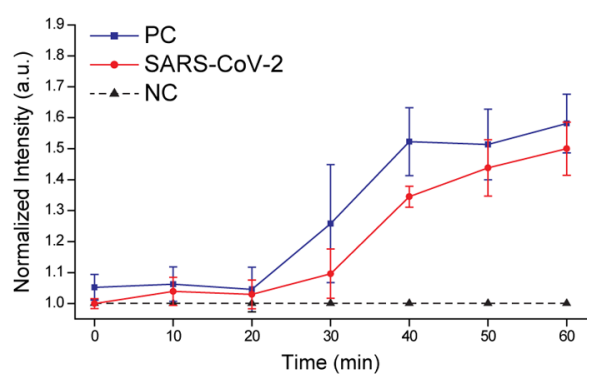

Figure 3. Autonomous RNA amplification and colorimetric detection. (a) Schematic showing the functions of individual components of the IH module. The exothermic reaction of $\mathrm{CaO}$ with water produces heat, and the melting of the wax regulates the temperature of the device to the desired range for the RNA amplification. (b) Measured temperature of the device with (red) and without (black) the wax following the onset of the exothermic reaction. The melting of the wax regulates the device temperature at $\sim 68-72{ }^{\circ} \mathrm{C}$ and prevented a temperature spike that would inactivate the Bst polymerase. (c) Measured device temperature loaded with different amounts of $\mathrm{CaO}$. $\mathrm{CaO}(5 \mathrm{~g})$ could maintain an isothermal environment at $\sim 68{ }^{\circ} \mathrm{C}$ for $>90 \mathrm{~min}$. (d) Images of the detection spots taken at different timepoints showing the changes in the spot colors as the reaction progresses. The images show the color on PC and SARS-CoV-2 spots changing from pink to yellow after 40 min, while the color on NC spot remains pink for the whole tested duration. (e) Measured color intensity of the PC, NC, and SARS-CoV-2 spots as functions of the reaction time. Error bars in all panels represent the standard deviation $(n=3)$.

chamber with a candelilla wax that melts at $\sim 68-72{ }^{\circ} \mathrm{C}$. Melting of the wax was confirmed to not only rectify temperature spikes but also store the heat to be dissipated at the desired temperature (Figure $3 \mathrm{~b}$ ). With this setup, it took $\sim 10 \mathrm{~min}$ to reach the desired temperature range, which was well before the 20 min mark for the arrival of RT-LAMP buffer at the detection spot. Furthermore, the isothermal conditions could be maintained for the desired duration depending on the starting amount of $\mathrm{CaO}$ in the reaction chamber-for example, $5 \mathrm{~g}$ of $\mathrm{CaO}$ powder was found to produce $\sim 68^{\circ} \mathrm{C}$ for $>90 \mathrm{~min}$ (Figure 3c). As a functional test, we employed the designed $\mathrm{IH}$ module to perform a conventional tube-based RT-LAMP reaction and confirmed successful RNA amplification (Figure S2).

Next, we investigated the colorimetric output of the assay to determine the amount of time required for the RNA amplification to produce a signal that could reliably be detected. Saliva samples $(20 \mu \mathrm{L})$ spiked with 1000 copies $(50$ copies $/ \mu \mathrm{L}$ ) of SARS-CoV-2 were processed on the complete assay, and the gradual color changes on the SARS-CoV-2 detection spot could visually be observed over time (Figure $3 \mathrm{~d}$ ). Beyond $40 \mathrm{~min}$, the SARS-CoV-2 spot color changed from pink to yellow, as well as the PC spot color. On the other hand, the NC spot remained to be pink with no observable color changes validating the amplification reaction as the source of the colorimetric signal. The amplification reaction on each spot was independently verified by adding a fluorescent intercalating dye to the reaction (Figure S3). Normalized color intensities at the PC, NC, and SARS-CoV-2 spots were calculated from camera images via digital image processing (see the Materials and Methods) (Figure 3e). The measurements showed that the colors began changing after $20 \mathrm{~min}$ right when the RNA amplification started with the arrival of the RT-LAMP buffer. The largest change in the colorimetric signal was between 20 and $40 \mathrm{~min}$ followed by increasingly diminishing gains. Based on these results, we concluded 60 min to be sufficiently long to reliably read the assay results.

Multiplex Detection of SARS-CoV-2 and Influenza A and B Viruses. Next, we tested using the optimized POC toolkit and the assay protocol for multiplex detection of SARSCoV-2 and influenza A and B viruses in saliva samples. Samples spiked with controlled amounts of either one of these virus types were processed on the device with no external interference except for depositing the saliva sample, reagents, and water into the designated ports at the onset. To determine the limit of detection (LoD) for the assay, we processed samples with virus concentrations varying from 0.5 to 5000 copies $/ \mu \mathrm{L}$ ( 10 to $10^{5}$ copies per $20 \mu \mathrm{L}$ of saliva) and evaluated color changes in the detection spots after $60 \mathrm{~min}$ (Figure 4a). For all samples tested, a concentration of 0.5 copies $/ \mu \mathrm{L}$ was found to be below the LoD of our assay as the detection spot color was indistinguishable from that of the NC. Although a concentration of 5 copies $/ \mu \mathrm{L}$ for all virus types on average produced detectable color changes $(\sim 7.4-12.7 \%)$, results showed a relatively high variation between measurements with a coefficient of variation (CV) of $\sim 0.61-0.74$ (Figure $4 \mathrm{~b}$ ). Assuming this statistical spread of the data to be due to the noise in our assay, we chose to define results with a $\mathrm{CV}$ of $<$ 
a

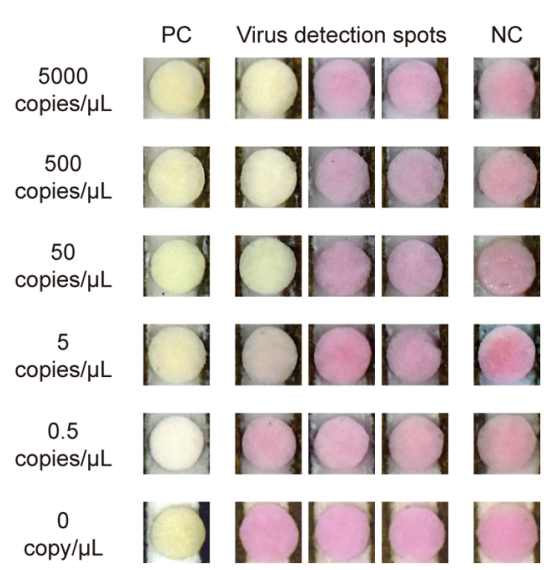

Influenza A test

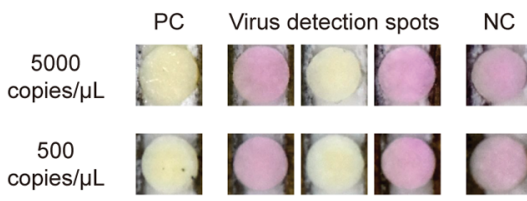

50

copies/ $/ \mathrm{L}$

5
copies/ $/ \mu \mathrm{L}$

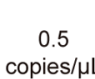

0.5
copies/uL

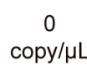

Influenza B test
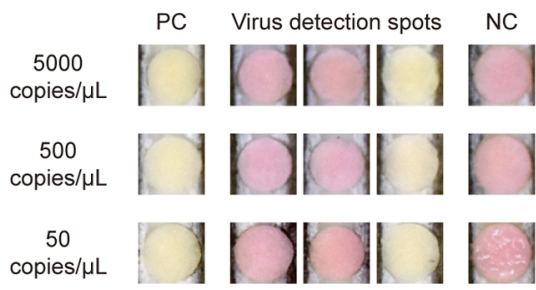

5

copies/ $\mu \mathrm{L}$

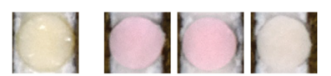

0.5

copies/ $\mu \mathrm{L}$

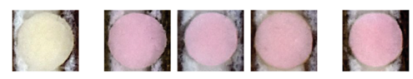

0

copy/uL
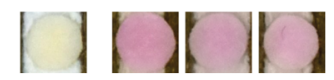

b
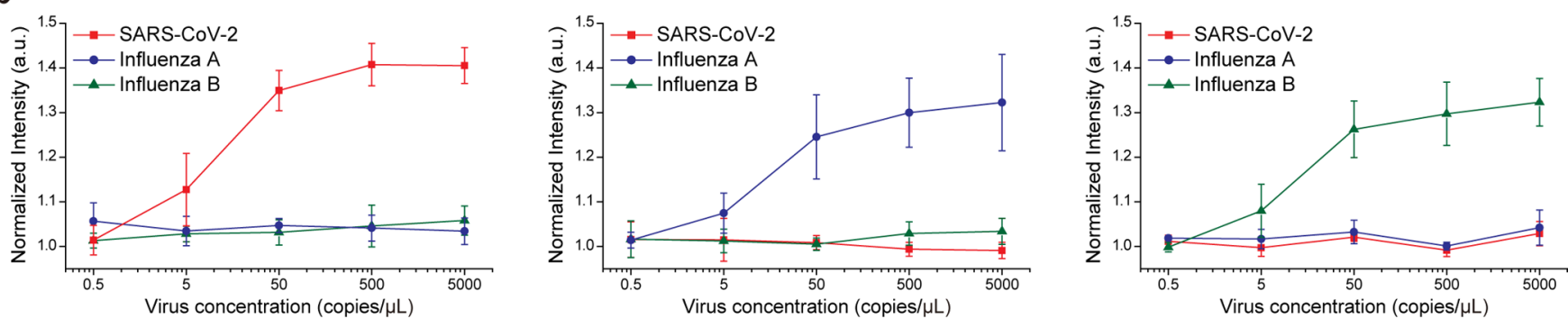

Figure 4. Multiplex detection of SARS-CoV-2 and influenza A and B viruses. (a) Assay results corresponding to the saliva samples spiked with different copy numbers of SARS-CoV-2, influenza A, and influenza B viruses. Among the three central detection spots, the left one tests for the presence of SARS-CoV-2, the middle one tests for influenza A, and the right spot tests for the influenza B virus. The change in the color of a spot indicates a positive result for the targeted virus. (b) Plots below the images show the measured color intensities for each test as functions of virus copy number. Error bars represent the standard deviation $(n=3)$.

0.33 as reliably detectable virus concentration levels when determining the assay LoD. Consequently, we identified 50 copies $/ \mu \mathrm{L}$ to be the LoD for our assay, which consistently produced a drastic change in the color intensity $(\sim 25-35 \%)$ with a CV of $\sim 0.13-0.32$ (Figure $4 \mathrm{~b}$ ). Further increases in virus concentration beyond 50 copies $/ \mu \mathrm{L}$ produced higher signals but with relatively smaller increases in the colorimetric signal levels.

We also found our assay to be successful in discriminating SARS-CoV-2 from the influenza $A$ and $B$ viruses. The colorimetric signals were produced exclusively from the detection spots corresponding to the target virus type with no noticeable cross-talk between detection spots reserved for a different virus type (Figure $4 \mathrm{~b}$ ). Furthermore, experiments with control samples with no spiked viruses did not produce false positive signals, further validating the specificity of our assay. These results also demonstrate the feasibility of scaling the technology for multiplex detection of more virus types or even different strains of SARS-CoV-2 on the same assay.

Application of the Assay on COVID-19 Patient Samples. Following the characterization of our assay with simulated virus-spiked saliva samples, we finally applied our assay to test saliva samples collected from COVID-19 patients. Processed patient samples $(n=3)$ were each independently tested by the vendor via RT-qPCR and confirmed to be positive for SARS-CoV-2 (Table S2). In agreement with these data, our assay was able to detect SARS-CoV-2 in all tested patient samples with color changes clearly visible on the detection spot dedicated to the detection of SARS-CoV-2
(Figure 5a). Furthermore, patient samples produced virtually no cross-talk as the mean normalized signal intensities measured on the detection spots reserved for the influenza A and B viruses were $\sim 2.0$ and $2.4 \%$, respectively, while the average normalized signal from the SARS-CoV-2 was $\sim 33.1 \%$ (Figure 5b).

To test the specificity of the assay, we also processed saliva samples collected from healthy donors $(n=3)$ according to Georgia IRB-approved protocols along with the patient samples. These control samples were all negative for SARSCoV-2 and influenza $\mathrm{A}$ and $\mathrm{B}$ viruses producing mean normalized intensity changes of $\sim 1.3, \sim 1.4$, and $\sim 1 \%$, respectively. Taken together, these results confirmed the validity of our assay and demonstrated the feasibility of applying the technology on actual patient saliva samples for the multiplexed molecular detection of SARS-CoV-2 and influenza $A$ and $B$ viruses.

\section{CONCLUSIONS}

Symptoms due to COVID-19 could easily be mixed with those from the common flu. We developed a POC assay that can reliably perform multiplex molecular detection of SARS-CoV-2 and influenza $\mathrm{A}$ and $\mathrm{B}$ viruses down to 50 copies $/ \mu \mathrm{L}$ in human saliva samples. Despite the purposely frugal nature of the assay presented in this work, multistep, multireagent chemical reactions for virus RNA extraction, purification, and amplification could all be autonomously performed within the device without compromising on the sensitivity or specificity of any of these reactions. Considering the SARS- 
a Positive samples

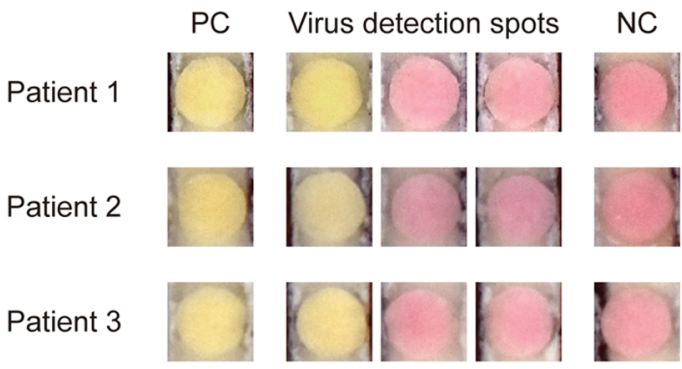

b

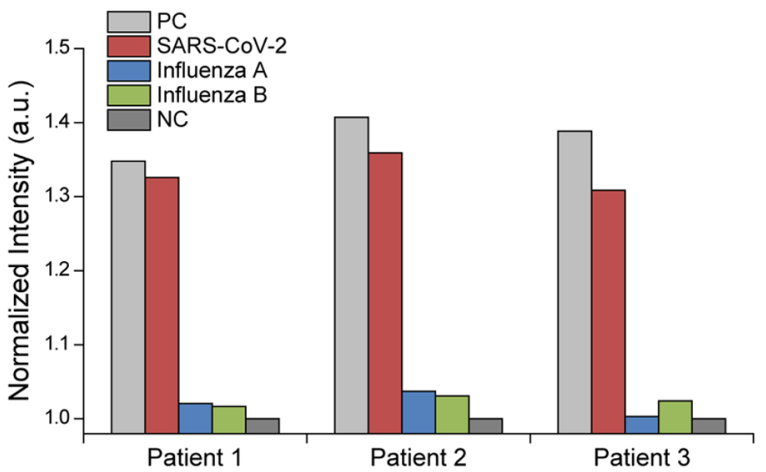

Negative samples
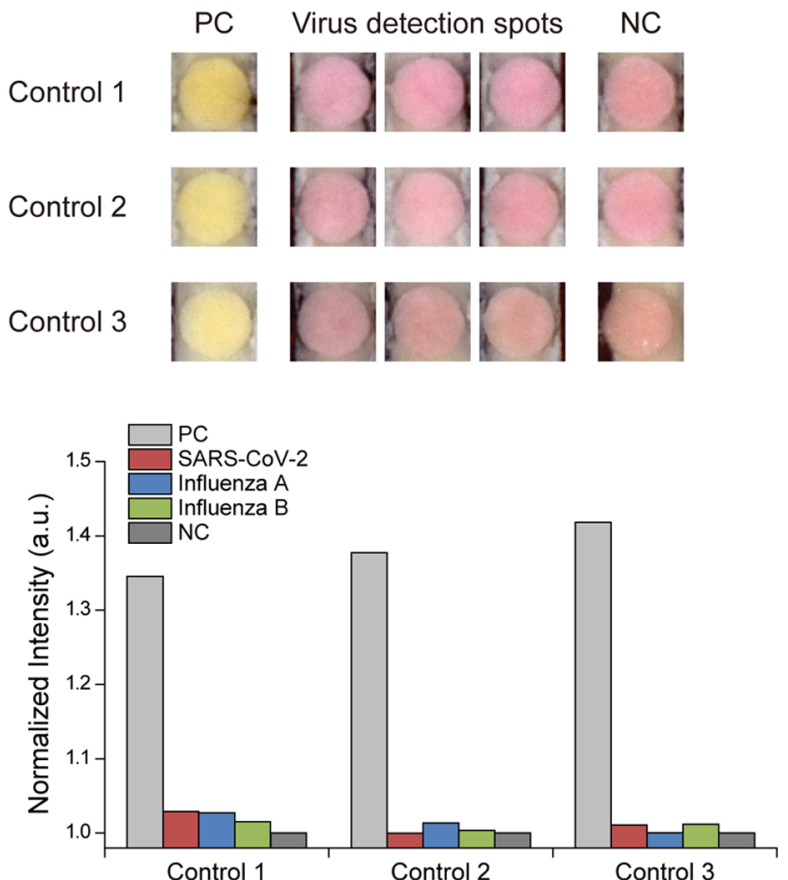

Figure 5. Application of the assay on COVID-19 patient samples. (a) Assay results corresponding to the saliva samples from three different COVID-19 patients (left) and healthy donors as controls (right). The leftmost and rightmost spots on the assay are PCs and NCs, respectively. Among the three central detection spots, the left one tests for the presence of SARS-CoV-2, the middle one tests for influenza A, and the right spot tests for the influenza B virus. The change in the color of the left spot for patient samples indicates a positive result for the SARS-CoV-2. (b) Plots below the images show the corresponding normalized color intensities for each spot for all tested saliva samples from COVID-19 patients (left) and healthy donors (right).

CoV-2 still threatening our lives with an increasing number of new infections every day, our work has the potential to contribute to the efforts in addressing the critical need for POC testing to positively identify COVID-19 infections by simplifying conventionally complex and labor-intensive genetic assays.

\section{MATERIALS AND METHODS}

Fabrication of the Extraction and Detection Module (ED Module). To fabricate the first and second paper layers, $25 \times 45 \mathrm{~mm}$ of the tissue paper (Kimtech Science Kimwipes delicate task wipers, Kimberly-Clark, Irving, TX) was immersed in a blocking buffer $[0.25 \%(\mathrm{v} / \mathrm{v})$ Tween-20 and $5 \%(\mathrm{w} / \mathrm{v})$ bovine serum albumin in phosphate-buffered saline (PBS)] for $1 \mathrm{~h}$. After washing in PBS and fully drying, one side of the tissue paper was covered with clear tape, followed by line drawing using an automatic drawing machine (Silhouette CAMEO, Silhouette America, Lindon, Utah) with two water-insoluble inks. A Sharpie metallic permanent marker was used for drawing the channel boundaries. A Sharpie ultrafine point marker was used to create timers. Each of $5 \mu \mathrm{L}$ primer mixtures targeting SARS-CoV-2 and influenza A and B viruses was dropped and dried on the assigned locations in the second paper layers. The double-sided tape was then attached for aligning and bonding the first and second layer, and five holes as the location of detection spots were punched using a $2.5 \mathrm{~mm}$ biopsy punch. To prepare the detection spots, five spots were punched out from a Whatman FTA Elute Card using a 2.5 $\mathrm{mm}$ biopsy punch. Three spots were placed in the central three holes in the aligned layers. The two remaining spots were washed and located in the remaining holes at both ends, serving as PC and NC. The SARS-CoV-2 PC template (N gene, 1000 copies $/ \mu \mathrm{L}, 1 \mu \mathrm{L}$ ) and SARS-CoV-2 primer (New England BioLabs, MA) were predried on the PC spot. The primer mixtures targeting SARS-CoV-2 and influenza A and B viruses were dried on the NC spot. After placing the detection spots in the designated locations, the device was sealed with clear tape on the top (with four inlet holes) and bottom.

Capillary Flow Control in Paper. The capillary flow on a laminated paper can be controlled by drawing patterns on the paper with two water-insoluble inks with different levels of hydrophobicity. ${ }^{38}$ The region patterned with a more hydrophobic ink (Sharpie metallic permanent marker) is permanently tethered to the sheath tape with no void formation, acting as channel boundaries to guide the flow. The region patterned with the less hydrophobic ink (Sharpie ultra fine point marker) eventually separates from the sheath tape when wetted, leaving the void to be formed at the interface between the patterned region and the tape. The flow is delayed until the void becomes large enough to allow the flow to proceed, acting as a timer. By imprinting and modifying the timer geometry (e.g., thickness or number) at strategic nodes, different flow delays are assigned to different reagents, enabling sequential flow into the desired locations following a programmed sequence.

Fabrication of the Internal Heat Module (IH Module). All of the IH module components were designed using Solidworks software (SolidWorks Corp., Waltham, MA). The dimensions of the individual components designed to form the $\mathrm{IH}$ module can be seen in Figure S4. These designs were printed using a 3D printer (ProJet $3510 \mathrm{HD}$ ) with VisiJet M3-X as a structural material. To dewax the uncured supporting material, the printed components were soaked in mineral oil (Durvet, Blue Springs, MO) at $65^{\circ} \mathrm{C}$ with sonication for $1 \mathrm{~h}$ and washed with soapy water, DI water, and ethanol, sequentially. After drying, the filler primer (Rust-Oleum, Automotive Filler Primer Spray) was sprayed on the components to render the surface smooth. After drying for $10 \mathrm{~min}$, white paint (Rust-Oleum, Painters Touch 2X Spray Paint Matte White) was sprayed on them and fully dried. The pellets of candelilla wax $\left(T_{\mathrm{m}}=68-72{ }^{\circ} \mathrm{C}, 15 \mathrm{~g}\right)$ were melted in a laboratory oven at $80^{\circ} \mathrm{C}$, and the melted wax was poured into the wax 
reservoir in the $\mathrm{IH}$ module. After solidifying the wax at room temperature, the strip of cellulose paper was located across the reservoirs of water and $\mathrm{CaO}$ powder, and $5 \mathrm{~g}$ of $\mathrm{CaO}$ powder was loaded into the center reservoir.

Characterization of Commercial RNA Sampling Papers. To compare the RNA extraction performance and compatibility with RTLAMP, three commercial nucleic-acid sampling papers [Whatman FTA Card, Whatman FTA Elute Card (GE Healthcare, UK), and RNASound RNA Sampling cards (FortiusBio LLC, CA)] were tested with cellulose and nitrocellulose paper as controls. Saliva samples (50 $\mu \mathrm{L})$ spiked with an inactivated SARS-CoV-2 intact virus particle were applied onto each paper, and the samples were air-dried for more than $3 \mathrm{~h}$. The dried samples on paper were punched out using a $2.5 \mathrm{~mm}$ biopsy punch, and each of them was placed into sterile microcentrifuge tubes for the following washing steps. The punched discs were then triple-washed in $500 \mu \mathrm{L}$ of DI water through gentle vortexing and centrifugation. Following the final wash, the washed discs were used for real-time RT-LAMP which was conducted on a QuantStudio 6 Flex real-time PCR system (Applied Biosystems, Foster City, CA). Whenever the reaction was conducted, the PC and NC were operated together.

Preparation of Real-Time/Colorimetric RT-LAMP Buffer. The reaction buffer for real-time/colorimetric RT-LAMP was obtained from a SARS-CoV-2 Rapid Colorimetric LAMP Assay Kit (New England BioLabs, MA). For real-time RT-LAMP, the buffer consists of $12.5 \mu \mathrm{L}$ of WarmStart colorimetric LAMP $2 \mathrm{X}$ master mix with UDG, $2.5 \mu \mathrm{L}$ of guanidine hydrochloride $(0.4 \mathrm{M}), 2.5 \mu \mathrm{L}$ of the primer mixture, $3.5 \mu \mathrm{L}$ of nuclease-free water, and $2 \mu \mathrm{L}$ of fluorescent intercalating dye $(1 \mu \mathrm{M}$, SYTO 9 green fluorescent nucleic acid stain, ThermoFisher Scientific, MA). Either $2 \mu \mathrm{L}$ of SARS-CoV-2 PC or nuclease-free water was added to the prepared buffer for PC and NC reaction. For testing RNA sampling papers, the punched papers and an additional $2 \mu \mathrm{L}$ of nuclease-free water were added to the prepared buffer. For colorimetric RT-LAMP, the buffer consists of $12.5 \mu \mathrm{L}$ of WarmStart colorimetric LAMP $2 \mathrm{X}$ master mix with UDG, $2.5 \mu \mathrm{L}$ of guanidine hydrochloride $(0.4 \mathrm{M})$, and $10 \mu \mathrm{L}$ of nuclease-free water. The principle for colorimetric detection is based on sensing $\mathrm{pH}$ change. The protons are released during the RT-LAMP as the byproducts and are accumulated in the buffer solution, which decreases $\mathrm{pH}$ significantly. The $\mathrm{pH}$ decrease in the buffer can visually be detected using $\mathrm{pH}$-sensitive dyes (e.g., phenol red). ${ }^{39,40}$

Primer Mixtures Targeting SARS-CoV-2 and Influenza A and B Viruses. The primer mixture targeting SARS-CoV-2 was obtained from the SARS-CoV-2 Rapid Colorimetric LAMP Assay Kit. The primer sequences targeting influenza $\mathrm{A}$ and $\mathrm{B}$ viruses were referred to previous literature ${ }^{41,42}$ and synthesized by oligo synthesis service (ThermoFisher Scientific, CA); each primer concentration was $8 \mu \mathrm{M}$ forward/reverse inner primers, $4 \mu \mathrm{M}$ loop primers, and $2 \mu \mathrm{M}$ forward/reverse outer primers. Each primer mixture $(5 \mu \mathrm{L})$ was dried on the assigned locations in the second paper layer. All primer sequences are listed in Table S1.

Virus Sample Preparation. The inactivated viruses of SARSCoV-2 (NATtrol SARS-CoV-2 External Run Controls) and influenza A (NATtrol Influenza A H1N1 External Run Controls) and B (NATtrol Influenza B External Run Controls), which have been chemically modified to render them noninfectious but are intact virus particles, were purchased from ZeptoMetrix Corporation (Buffalo, NY, USA). Saliva samples were collected from healthy donors according to protocols approved by IRB of Georgia Institute of Technology. To estimate the virus copy number in samples, each undiluted virus sample was spiked to the collected saliva and the spiked samples were processed using the ED module. The detection spots were taken out from the device using a tweezer, and the extracted viral RNA was eluted in $20 \mu \mathrm{L}$ of nuclease-free water. The concentration of the eluted RNA was quantified using a NanoDrop 1000 spectrophotometer (ThermoFisher Scientific, Wilmington, DE, USA) and converted to copy number based on RNA genome size of each virus: $30 \mathrm{~kb}$ for SARS-CoV-2, $13.5 \mathrm{~kb}$ for influenza $\mathrm{A}$, and 14.5 $\mathrm{kb}$ for influenza $\mathrm{B}$ virus. Then, the spiked samples were serially diluted to control the virus concentration $(5000-0.5$ copies $/ \mu \mathrm{L})$. PCR- confirmed COVID-19 patient saliva samples were obtained from Lee Biosolutions, Inc. (Maryland Heights, MO, USA).

Quantification of Color Intensity. The images of the five detection spots were captured after the RT-LAMP reaction and were individually analyzed using the ImageJ program (http://imagej.nih. gov/ij/). The raw images were split into separate RGB channels, and the green channel image was used for quantification; the green is a complementary color of pink-colored RT-LAMP buffer. Measured color intensities in each spot were divided by the intensity in the NC spot for normalization.

\section{ASSOCIATED CONTENT}

\section{(s) Supporting Information}

The Supporting Information is available free of charge at https://pubs.acs.org/doi/10.1021/acssensors.1c00702.

Testing of the covered assay for possible evaporation of reagents during the amplification process; functional testing of the IH module; testing of the RNA amplification reaction with fluorescence measurements; detailed design and dimensions of the IH module; primer sequences targeting SARS-CoV-2 and influenza A and $\mathrm{B}$ viruses; and comparison of vendor-provided RTqPCR with the results from our assay for each patient sample tested (PDF)

\section{AUTHOR INFORMATION}

\section{Corresponding Author}

A. Fatih Sarioglu - School of Electrical and Computer Engineering, Georgia Institute of Technology, Atlanta, Georgia 30332, United States; Parker H. Petit Institute for Bioengineering and Bioscience and Institute for Electronics and Nanotechnology, Georgia Institute of Technology, Atlanta, Georgia 30332, United States; 이이. orcidorgo00001-7191-4342; Email: sarioglu@gatech.edu

\section{Authors}

Dohwan Lee - School of Electrical and Computer Engineering, Georgia Institute of Technology, Atlanta, Georgia 30332, United States

Chia-Heng Chu - School of Electrical and Computer Engineering, Georgia Institute of Technology, Atlanta, Georgia 30332, United States

Complete contact information is available at: https://pubs.acs.org/10.1021/acssensors.1c00702

\section{Author Contributions}

D.L. and A.F.S. designed the research and overall direction; D.L. and C.-H.C. fabricated the devices; D.L. performed experiments; all authors conducted data analysis; D.L. and A.F.S. wrote the manuscript; and all authors discussed and commented on refining the final manuscript.

\section{Notes}

The authors declare no competing financial interest.

\section{ACKNOWLEDGMENTS}

This work was supported by the start-up funds provided to A.F.S. by Georgia Institute of Technology.

\section{REFERENCES}

(1) Tian, H.; Liu, Y.; Li, Y.; Wu, C.-H.; Chen, B.; Kraemer, M. U. G.; Li, B.; Cai, J.; Xu, B.; Yang, Q.; et al. An Investigation of Transmission Control Measures during the First 50 Days of the COVID-19 Epidemic in China. Science 2020, 368, 638-642. 
(2) Zhang, J.; Litvinova, M.; Liang, Y.; Wang, Y.; Wang, W.; Zhao, S.; Wu, Q.; Merler, S.; Viboud, C.; Vespignani, A.; et al. Changes in Contact Patterns Shape the Dynamics of the COVID-19 Outbreak in China. Science 2020, 368, 1481-1486.

(3) Salje, H.; Tran Kiem, C.; Lefrancq, N.; Courtejoie, N.; Bosetti, P.; Paireau, J.; Andronico, A.; Hozé, N.; Richet, J.; Dubost, C.-L.; et al. Estimating the Burden of SARS-CoV-2 in France. Science 2020, 369, 208-211.

(4) Chinazzi, M.; Davis, J. T.; Ajelli, M.; Gioannini, C.; Litvinova, M.; Merler, S.; Pastore y Piontti, A.; Mu, K.; Rossi, L.; Sun, K.; et al. The Effect of Travel Restrictions on the Spread of the 2019 Novel Coronavirus (COVID-19) Outbreak. Science 2020, 368, 395-400.

(5) Menni, C.; Valdes, A. M.; Freidin, M. B.; Sudre, C. H.; Nguyen, L. H.; Drew, D. A.; Ganesh, S.; Varsavsky, T.; Cardoso, M. J.; ElSayed Moustafa, J. S.; et al. Real-Time Tracking of Self-Reported Symptoms to Predict Potential COVID-19. Nat. Med. 2020, 26, 1037-1040.

(6) Liu, Y.; Ning, Z.; Chen, Y.; Guo, M.; Liu, Y.; Gali, N. K.; Sun, L.; Duan, Y.; Cai, J.; Westerdahl, D.; et al. Aerodynamic Analysis of SARS-CoV-2 in Two Wuhan Hospitals. Nature 2020, 582, 557-560.

(7) Wölfel, R.; Corman, V. M.; Guggemos, W.; Seilmaier, M.; Zange, S.; Müller, M. A.; Niemeyer, D.; Jones, T. C.; Vollmar, P.; Rothe, C.; et al. Virological Assessment of Hospitalized Patients with COVID2019. Nature 2020, 581, 465-469.

(8) Carter, L. J.; Garner, L. V.; Smoot, J. W.; Li, Y.; Zhou, Q.; Saveson, C. J.; Sasso, J. M.; Gregg, A. C.; Soares, D. J.; Beskid, T. R.; et al. Assay Techniques and Test Development for COVID-19 Diagnosis. ACS Cent. Sci. 2020, 6, 591-605.

(9) van Kasteren, P. B.; van der Veer, B.; van den Brink, S.; Wijsman, L.; de Jonge, J.; van den Brandt, A.; Molenkamp, R.; Reusken, C. B. E. M.; Meijer, A. Comparison of Seven Commercial RT-PCR Diagnostic Kits for COVID-19. J. Clin. Virol. 2020, 128, 104412.

(10) Udugama, B.; Kadhiresan, P.; Kozlowski, H. N.; Malekjahani, A.; Osborne, M.; Li, V. Y. C.; Chen, H.; Mubareka, S.; Gubbay, J. B.; Chan, W. C. W. Diagnosing COVID-19: The Disease and Tools for Detection. ACS Nano 2020, 14, 3822-3835.

(11) Centers for Disease Control and Prevention. CDC's Diagnostic Multiplex Assay for Flu and COVID-19 at Public Health Laboratories and Supplies. 2020, https://www.cdc.gov/coronavirus/2019-ncov/ lab/multiplex.html.

(12) Zhao, Y.; Chen, F.; Li, Q.; Wang, L.; Fan, C. Isothermal Amplification of Nucleic Acids. Chem. Rev. 2015, 115, 12491-12545.

(13) Qian, J.; Boswell, S. A.; Chidley, C.; Lu, Z.-x.; Pettit, M. E.; Gaudio, B. L.; Fajnzylber, J. M.; Ingram, R. T.; Ward, R. H.; Li, J. Z.; et al. An Enhanced Isothermal Amplification Assay for Viral Detection. Nat. Commun. 2020, 11, 5920.

(14) Becherer, L.; Borst, N.; Bakheit, M.; Frischmann, S.; Zengerle, R.; Von Stetten, F. Loop-Mediated Isothermal Amplification (LAMP)-Review and Classification of Methods for Sequence-Specific Detection. Anal. Methods 2020, 12, 717-746.

(15) Liu, W.; Dong, D.; Yang, Z.; Zou, D.; Chen, Z.; Yuan, J.; Huang, L. Polymerase Spiral Reaction (PSR): A Novel Isothermal Nucleic Acid Amplification Method. Sci. Rep. 2015, 5, 12723.

(16) Gill, P.; Ghaemi, A. Nucleic Acid Isothermal Amplification Technologies - A Review. Nucleosides, Nucleotides Nucleic Acids 2008, 27, 224-243.

(17) Notomi, T.; Okayama, H.; Masubuchi, H.; Yonekawa, T.; Watanabe, K.; Amino, N.; Hase, T. Loop-Mediated Isothermal Amplification of DNA. Nucleic Acids Res. 2000, 28, No. e63.

(18) El-Tholoth, M.; Bai, H.; Mauk, M. G.; Saif, L.; Bau, H. H. A Portable, 3D Printed, Microfluidic Device for Multiplexed, Real Time, Molecular Detection of the Porcine Epidemic Diarrhea Virus, Transmissible Gastroenteritis Virus, and Porcine Deltacoronavirus at the Point of Need. Lab Chip 2021, 21, 1118-1130.

(19) Agranovski, I. E.; Usachev, E. V. In-Situ Rapid Bioaerosol Detection in the Ambient Air by Miniature Multiplex PCR Utilizing Technique. Atmos. Environ. 2021, 246, 118147.

(20) Rizzo, M. G.; Carnazza, S.; De Plano, L. M.; Franco, D.; Nicolò, M. S.; Zammuto, V.; Petralia, S.; Calabrese, G.; Gugliandolo, C.;
Conoci, S.; et al. Rapid Detection of Bacterial Pathogens in Blood through Engineered Phages-Beads and Integrated Real-Time PCR into MicroChip. Sens. Actuators, B 2021, 329, 129227.

(21) Trinh, T. N. D.; Lee, N. Y. A Foldable Isothermal Amplification Microdevice for Fuchsin-Based Colorimetric Detection of Multiple Foodborne Pathogens. Lab Chip 2019, 19, 1397-1405.

(22) Lee, D.; Kim, Y. T.; Lee, J. W.; Kim, D. H.; Seo, T. S. An Integrated Direct Loop-Mediated Isothermal Amplification Microdevice Incorporated with an Immunochromatographic Strip for Bacteria Detection in Human Whole Blood and Milk without a Sample Preparation Step. Biosens. Bioelectron. 2016, 79, 273-279.

(23) Lee, D.; Shin, Y.; Chung, S.; Hwang, K. S.; Yoon, D. S.; Lee, J. H. Simple and Highly Sensitive Molecular Diagnosis of Zika Virus by Lateral Flow Assays. Anal. Chem. 2016, 88, 12272-12278.

(24) Connelly, J. T.; Rolland, J. P.; Whitesides, G. M. "Paper Machine” for Molecular Diagnostics. Anal. Chem. 2015, 87, 75957601.

(25) Barza, R.; Patel, P.; Sabatini, L.; Singh, K. Use of a Simplified Sample Processing Step without RNA Extraction for Direct SARSCoV-2 RT-PCR Detection. J. Clin. Virol. 2020, 132, 104587.

(26) Fukumoto, T.; Iwasaki, S.; Fujisawa, S.; Hayasaka, K.; Sato, K.; Oguri, S.; Taki, K.; Nakakubo, S.; Kamada, K.; Yamashita, Y.; et al. Efficacy of a Novel SARS-CoV-2 Detection Kit without RNA Extraction and Purification. Int. J. Infect. Dis. 2020, 98, 16-17.

(27) Taki, K.; Yokota, I.; Fukumoto, T.; Iwasaki, S.; Fujisawa, S.; Takahashi, M.; Negishi, S.; Hayasaka, K.; Sato, K.; Oguri, S.; Nishida, M.; Sugita, J.; Konno, S.; Saito, T.; Teshima, T. SARS-CoV-2 Detection by Fluorescence Loop-Mediated Isothermal Amplification with and without RNA Extraction. J. Infect. Chemother. 2021, 27, 410-412.

(28) Zhang, H.; Xu, Y.; Fohlerova, Z.; Chang, H.; Iliescu, C.; Neuzil, P. LAMP-on-a-Chip: Revising Microfluidic Platforms for LoopMediated DNA Amplification. Trends Anal. Chem. 2019, 113, 44-53. (29) Seok, Y.; Joung, H.-A.; Byun, J.-Y.; Jeon, H.-S.; Shin, S. J.; Kim, S.; Shin, Y.-B.; Han, H. S.; Kim, M.-G. A Paper-Based Device for Performing Loop-Mediated Isothermal Amplification with Real-Time Simultaneous Detection of Multiple DNA Targets. Theranostics 2017, 7, 2220-2230.

(30) Kaarj, K.; Akarapipad, P.; Yoon, J.-Y. Simpler, Faster, and Sensitive Zika Virus Assay Using Smartphone Detection of LoopMediated Isothermal Amplification on Paper Microfluidic Chips. Sci. Rep. 2018, 8, 12438.

(31) Kaur, N.; Toley, B. J. Paper-Based Nucleic Acid Amplification Tests for Point-of-Care Diagnostics. Analyst 2018, 143, 2213-2234.

(32) Choi, J. R.; Hu, J.; Gong, Y.; Feng, S.; Wan Abas, W. A. B.; Pingguan-Murphy, B.; Xu, F. An Integrated Lateral Flow Assay for Effective DNA Amplification and Detection at the Point of Care. Analyst 2016, 141, 2930-2939.

(33) Choi, J. R.; Hu, J.; Tang, R.; Gong, Y.; Feng, S.; Ren, H.; Wen, T.; Li, X.; Wan Abas, W. A. B.; Pingguan-Murphy, B.; et al. An Integrated Paper-Based Sample-to-Answer Biosensor for Nucleic Acid Testing at the Point of Care. Lab Chip 2016, 16, 611-621.

(34) Batule, B. S.; Seok, Y.; Kim, M.-G. Paper-Based Nucleic Acid Testing System for Simple and Early Diagnosis of Mosquito-Borne RNA Viruses from Human Serum. Biosens. Bioelectron. 2020, 151, 111998.

(35) Paul, R.; Ostermann, E.; Chen, Y.; Saville, A. C.; Yang, Y.; Gu, Z.; Whitfield, A. E.; Ristaino, J. B.; Wei, Q. Integrated MicroneedleSmartphone Nucleic Acid Amplification Platform for in-Field Diagnosis of Plant Diseases. Biosens. Bioelectron. 2021, 187, 113312.

(36) De Oliveira, K. G.; Estrela, P. F. N.; Mendes, G. D. M.; Dos Santos, C. A.; Silveira-Lacerda, E. D. P.; Duarte, G. R. M. Rapid Molecular Diagnostics of COVID-19 by RT-LAMP in a Centrifugal Polystyrene-Toner Based Microdevice with End-Point Visual Detection. Analyst 2021, 146, 1178-1187.

(37) Rodriguez-Manzano, J.; Malpartida-Cardenas, K.; Moser, N.; Pennisi, I.; Cavuto, M.; Miglietta, L.; Moniri, A.; Penn, R.; Satta, G.; Randell, P.; et al. Handheld Point-of-Care System for Rapid Detection 
of SARS-CoV-2 Extracted RNA in under 20 Min. ACS Cent. Sci. 2021, 7, 307-317.

(38) Lee, D.; Ozkaya-Ahmadov, T.; Chu, C.-H.; Boya, M.; Liu, R.; Sarioglu, A. F. Capillary flow control in lateral flow assays via delaminating timers. Sci. Adv. 2021, 7, eabf9833 (in press).

(39) Tanner, N. A.; Zhang, Y.; Evans, T. C. Visual Detection of Isothermal Nucleic Acid Amplification Using PH-Sensitive Dyes. Biotechniques 2015, 58, 59-68.

(40) Zhang, Y.; Odiwuor, N.; Xiong, J.; Sun, L.; Nyaruaba, R. O.; Wei, H.; Tanner, N. Rapid Molecular Detection of SARS-CoV-2 (COVID-19) Virus RNA Using Colorimetric LAMP. 2020, medRxiv:2020.02.26.20028373. Preprint. https://www.medrxiv.org/ content/10.1101/2020.02.26.20028373v1.

(41) Kubo, T.; Agoh, M.; Mai, L. Q.; Fukushima, K.; Nishimura, H.; Yamaguchi, A.; Hirano, M.; Yoshikawa, A.; Hasebe, F.; Kohno, S.; et al. Development of a Reverse Transcription-Loop-Mediated Isothermal Amplification Assay for Detection of Pandemic (H1N1) 2009 Virus as a Novel Molecular Method for Diagnosis of Pandemic Influenza in Resource-Limited Settings. J. Clin. Microbiol. 2010, 48, $728-735$.

(42) Mahony, J.; Chong, S.; Bulir, D.; Ruyter, A.; Mwawasi, K.; Waltho, D. Multiplex Loop-Mediated Isothermal Amplification (MLAMP) Assay for the Detection of Influenza $\mathrm{A} / \mathrm{H} 1, \mathrm{~A} / \mathrm{H} 3$ and Influenza B Can Provide a Specimen-to-Result Diagnosis in 40min with Single Genome Copy Sensitivity. J. Clin. Virol. 2013, 58, 127131. 